\title{
Event-Triggered Consensus Control for Discrete-time Stochastic Multi-agent Systems: The Input-to-State Stability in Probability *
}

\author{
Derui Ding ${ }^{\mathrm{a}}$, Zidong Wang ${ }^{\mathrm{b}}$, Bo Shen ${ }^{\mathrm{c}}$, Guoliang Wei ${ }^{\mathrm{a}}$ \\ ${ }^{a}$ Shanghai Key Lab of Modern Optical System, Department of Control Science and Engineering, University of Shanghai for \\ Science and Technology, Shanghai 200093, China. \\ ${ }^{\mathrm{b}}$ Department of Computer Science, Brunel University London, Uxbridge, Middlesex, UB8 3PH, U.K. \\ ${ }^{\mathrm{c}}$ School of Information Science and Technology, Donghua University, Shanghai 200051, China.
}

\begin{abstract}
This paper is concerned with the event-triggered consensus control problem for a class of discrete-time stochastic multi-agent systems with state-dependent noises. A novel definition of consensus in probability is proposed to better describe the dynamics of the consensus process of the addressed stochastic multi-agent systems. The measurement output available for the controller is not only from the individual agent but also from its neighboring ones according to the given topology. An event-triggered mechanism is adopted with hope to reduce the communication burden, where the control input on each agent is updated only when a certain triggering condition is violated. The purpose of the problem under consideration is to design both the output feedback controller and the threshold of the triggering condition such that the closed-loop system achieves the desired consensus in probability. First of all, a theoretical framework is established for analyzing the so-called input-to-state stability in probability (ISSiP) for general discrete-time nonlinear stochastic systems. Within such a theoretical framework, some sufficient conditions on event-triggered control protocol are derived under which the consensus in probability is reached. Furthermore, both the controller parameter and the triggering threshold are obtained in terms of the solution to certain matrix inequalities involving the topology information and the desired consensus probability. Finally, a simulation example is utilized to illustrate the usefulness of the proposed control protocol.
\end{abstract}

Key words: Multi-agent systemsConsensus in probability; Event-triggered control; Input-to-state stability in probability; Discrete-time stochastic nonlinear systems.

\section{Introduction}

The past decade has seen a surge of research interest on the consensus problems for multi-agent systems due to their extensive applications in various engineering systems such as cooperative control of unmanned air vehicles (UAVs) or unmanned underwater vehicles (UUVs), formation control for multi-robot systems, collective behaviors of flocks or swarms, distributed sensor networks, and attitude alignment of clusters of satellites. Up to now, there has been a rich body of results on consensus analysis issues for multi-agent systems with stochastic noises, communication delays, or limited communication data rate, see $[1,12]$ and the references therein.

A common assumption in almost all the aforemen-

\begin{abstract}
* This work was supported in part by Royal Society of the UK, the National Natural Science Foundation of China under Grants 61329301, 61203139 and 61473076, the Hujiang Foundation of China under Grants C14002 and D15009, the Shanghai Rising-Star Program of China under Grant 13QA1400100, the ShuGuang project of Shanghai Municipal Education Commission and Shanghai Education Development Foundation under Grant 13SG34, the Fundamental Research Funds for the Central Universities, DHU Distinguished Young Professor Program, and the Alexander von Humboldt Foundation of Germany.
\end{abstract}

Email addresses: deruiding2010@usst.edu.cn (Derui Ding), Zidong.Wang@brunel.ac.uk (Zidong Wang). tioned literature is that the consensus controllers are time-triggered rather than event-triggered. Nevertheless, from a technical viewpoint, the event-triggered control strategy is more attractive, especially the distributed real-time sensing and control due mainly to the need for reducing the communication burden [11]. On the other hand, in order to prolong the life time of digital control circuits, an effective method is to reduce the triggering frequency of electronic components. Therefore, the event-triggered scheduling seems to be more suitable for the consensus control of multi-agent systems and, accordingly, the event-triggered consensus control has received particular research attention in the past few years leading to some preliminary results reported in $[11,13,19]$. Note that most papers have been concerned with the asymptotic consensus issues for the 1st or 2nd order multi-agent systems with a relative change triggering condition, see Table 1 for a brief summary. Such a condition could result in the rendezvous phenomenon and increase the computational burden in comparison with the absolute change condition. In addition, these results are mainly based on state-feedback control for multi-agent systems without stochastic disturbances, and the desired controller gains have been obtained in the framework of Lyapunov stability theory. However, the corresponding results concerning outputfeedback control have been scattered in the literature for general multi-agent systems. 
Table 1

A brief summary of event-based consensus control

\begin{tabular}{|l|l|l|l|}
\hline \multirow{4}{*}{ Items } & \multirow{2}{|l|}{$\begin{array}{l}\text { Non-stochastic } \\
\text { systems }\end{array}$} & $\begin{array}{l}\text { The 1st or 2nd } \\
\text { order systems }\end{array}$ & {$[11,13]$} \\
\cline { 2 - 4 } Systems & General systems & {$[19]$} \\
\cline { 2 - 4 } & $\begin{array}{l}\text { Stochastic } \\
\text { systems }\end{array}$ & $\begin{array}{l}\text { The 1st or 2nd } \\
\text { order systems }\end{array}$ & {$[17]$} \\
\cline { 2 - 4 } & General systems & {$[7]$} \\
\hline \hline \multirow{2}{*}{$\begin{array}{l}\text { Triggering } \\
\text { conditions }\end{array}$} & Absolute change conditions & {$[13]$} \\
\cline { 2 - 4 } Performances & Relative change conditions & {$[11,17]$} \\
\hline \hline \multirow{2}{*}{\begin{tabular}{l} 
Asymptotic consensus \\
\cline { 2 - 4 }
\end{tabular}} & Bounded consensus & {$[7,11,17]$} \\
\hline \hline Design \\
parameters & Controller parameters & {$[13,19]$} \\
\cline { 2 - 4 } & Controller \& trigger parameters & {$[13,19]$} \\
\hline
\end{tabular}

Compared with the time-triggered control scheme $[2$, $3,15,16]$, the distinct feature of event-triggered control strategy is that the control input is updated only when certain specific event happens. Between updates of the controller inputs, the control signal remains unchanged, and an appropriately generated updating time would guarantee the stability and performance of the closed-loop system. It is worth noting that the updating time is typically unknown a priori since they are determined by the event triggering mechanisms dependent on time, sampling data, system states. Nowadays, three arguably representative theoretical frameworks, namely, Lyapunov stability theory, switched system theory and input-to-state stability (ISS) theory [10] have been widely employed to analyze the event-triggered control issues. In the ISS framework, the event triggering condition can be obtained by exploiting the distance measures between certain signals of interest, for example, the tracking error, the difference between the data at the current sampling instant and that at the latest transmission instant, and the difference between controller's updates. Recently, the discrete-time stochastic linear systems with event-triggered schemes have received particular research attention and some preliminary results have been reported in $[6]$ for stability analysis and linear-quadratic (LQ) control. However, the corresponding issues for more general discrete-time systems with stochastic disturbances have not gained adequate research attention due primarily to the difficulty in both the mathematical analysis and the design of suitable event triggering mechanisms. For instance, although significant progress has been made for the ISS theory of continuous-time stochastic nonlinear systems $[9,14]$, the results for their discrete-time counterparts have been very few, which constitutes the main motivation of our present investigation.

In reality, the challenges in designing event-triggered controllers for multi-agent systems stem mainly from proper definition of the consensus performance, the mathematical difficulty in consensus analysis and the effects from network topology and external disturbances. So far, most research focuses have been on the steadystate behaviors of the consensus, i.e., the consensus performance when time tends to infinity. Another important system behavior, namely, transient consensus performance at specific times, has been largely overlooked despite its significance in reflecting the consensus dynamics. On the other hand, rather than requiring the objectives of the system performance to be met accurately, it is quite common for multi-agent systems design to have their individual performance objective being described in terms of the desired probability of attaining that objective. In this sense, the concept of consensus in probability appears to be of engineering significance since 1) it is almost impossible to assure that certain performances are achieved with probability 1 because of uncontrolled external forces; and 2) in some cases, the commonly used mean-square measure might be crude to quantify the consensus performance and it would be satisfactory if certain performances are achieved with an acceptable probability [4]. It is, therefore, the main purpose of this paper to provide a systematic event-triggered design scheme ensuring the consensus in probability for a class of discrete-time stochastic multi-agent systems.

Motivated by the above discussions, in this paper, we aim to design an event-triggered controller for a class of discrete-time stochastic multi-agent systems with state dependent noises in order to guarantee the consensus performance in probability. The challenges we are going to cope with are identified as follows: 1) how can we establish a general analysis framework (i.e. discretetime version of stochastic ISS) to analyze the dynamical behaviors in probability of the multi-agent systems? 2) how can we design an appropriate threshold of the triggering condition based on the measurement outputs? 3) how can we examine the impact from the given topology and the consensus probability on the consensus performance? To handle the three identified challenges, by using the stochastic analysis techniques, we will first establish a discrete-time version of the input-to-state stability in probability (ISSiP), which plays an essential role in the performance analysis of discrete-time stochastic multi-agent systems. Within such a theoretical framework, both the output feedback controller and the threshold of the triggering condition are designed such that the closed-loop system achieves the desired consensus in probability.

The novelties of this paper lie in the following four aspects: 1) the model under consideration, which includes the commonly studied first- or second-order systems as its special cases, is quite comprehensive to describe discretetime stochastic multi-agent systems with state-dependent noise; 2) a novel definition of consensus in probability is proposed to account for the transient dynamics of the consensus process at certain specific time points with given consensus probability; 3) the discrete-time version of ISSiP for general discrete-time stochastic nonlinear systems is established in order to facilitate the later analysis of the consensus performance; and 4) both the output feedback controller and the threshold of the triggering condition are obtained by solving a set of parameterdependent matrix inequalities reflecting both the topology information and the desired consensus probability.

Notation The notation used here is fairly standard except where otherwise stated. $\mathbb{R}^{n}$ and $\mathbb{R}^{n \times m}$ denote, respectively, the $n$-dimensional Euclidean space and the set of all $n \times m$ real matrices. The set of all non-negative integers is denoted by $\mathbb{N}$. 1 denotes a compatible dimensional column vector with all ones. $I$ denotes the identity matrix of compatible dimensions. The notation $X \geq Y$ (respectively, $X>Y$ ), where $X$ and $Y$ are symmetric matrices, means that $X-Y$ is positive semi-definite (re- 
spectively, positive definite). $A^{T}$ represents the transpose of $A$. For matrices $A \in \mathbb{R}^{m \times n}$ and $B \in \mathbb{R}^{p \times q}$, their Kronecker product is a matrix in $\mathbb{R}^{m p \times n q}$ denoted as $A \otimes B .|x|$ describes the Euclidean norm of a vector $x$. $\operatorname{diag}\{\cdots\}$ stands for a block-diagonal matrix.

\section{Problem Formulation and Preliminaries}

In this paper, the multi-agent system has $N$ agents which communicate with each other according to a fixed network topology represented by an undirected graph $\mathscr{G}=(\mathscr{V}, \mathscr{E}, \mathscr{H})$ of order $N$ with the set of agents $\mathscr{V}=\{1,2, \cdots, N\}$, the set of edges $\mathscr{E} \in \mathscr{V} \times \mathscr{V}$, and the weighted adjacency matrix $\mathscr{H}=\left[h_{i j}\right]$ with nonnegative adjacency element $h_{i j}$. If $(i, j) \in \mathscr{E}$, then $h_{i j}>0$, else $h_{i j}=0$. An edge of $\mathscr{G}$ is denoted by the ordered pair $(i, j)$. The adjacency elements associated with the edges of the graph are positive, i.e., $h_{i j}>0 \Longleftrightarrow(i, j) \in \mathscr{E}$, which means that agent $i$ can obtain information from agent $j$. Furthermore, self-edges $(i, i)$ are not allowed, i.e. $(i, i) \notin \mathscr{E}$ for any $i \in \mathscr{V}$. The neighborhood of agent $i$ is denoted by $\mathscr{N}_{i}=\{j \in \mathscr{V}:(j, i) \in \mathscr{E}\}$. An element of $\mathscr{N}_{i}$ is called a neighbor of agent $i$. The in-degree of agent $i$ is defined as $\operatorname{deg}_{\text {in }}^{i}=\sum_{j \in \mathscr{N}_{i}} h_{i j}$.

Consider a discrete-time stochastic multi-agent system consisting of $N$ agents, in which the dynamics of agent $i$ is described by

$$
\left\{\begin{aligned}
x_{i}(k+1) & =A x_{i}(k)+D x_{i}(k) w(k)+B u_{i}(k) \\
y_{i}(k) & =C x_{i}(k)+E x_{i}(k) v(k)
\end{aligned}\right.
$$

where $x_{i}(k) \in \mathbb{R}^{n}, y_{i}(k) \in \mathbb{R}^{q}$ and $u_{i}(k) \in \mathbb{R}^{p}$ are, respectively, the states, the measurement outputs and the control inputs of agent $i . w(k), v(k) \in \mathbb{R}$ are zeromean random sequences with $\mathbb{E}\left\{w^{2}(k)\right\}=\mathbb{E}\left\{v^{2}(k)\right\}=1$ and $\mathbb{E}\{w(k) v(k)\}=\sigma^{2}$. A, B, C,D and $E$ are known constant matrices with compatible dimensions. It is assumed that the rank of $B$ is $p$.

In this paper, a control protocol with the following form is adopted:

$$
u_{i}(k)=K \sum_{j \in \mathscr{N}_{i}} h_{i j}\left(y_{j}(k)-y_{i}(k)\right):=K \phi_{i}(k)
$$

where $K \in \mathbb{R}^{n \times q}$ is a feedback gain matrix to be determined and $\phi_{i}(k)$ is the input signal of the controller which can be employed to describe the disagreement between agents' measurements.

In order to characterize the event triggering mechanism, let the triggering time sequence of agent $i$ be $0 \leq k_{0}^{i}<$ $k_{1}^{i}<k_{2}^{i}<\cdots<k_{s}^{i}<\cdots$ and the event generator functions $\Upsilon_{i}(\cdot, \cdot): \mathbb{R}^{q} \times \mathbb{R} \rightarrow \mathbb{R}(i=1,2, \cdots, N)$ be selected as follows:

$$
\Upsilon_{i}\left(e_{i}(k), \theta_{i}\right)=e_{i}^{T}(k) e_{i}(k)-\theta_{i}
$$

Here, $e_{i}(k):=\phi_{i}^{u}\left(k_{s}^{i}\right)-\phi_{i}(k)\left(k>k_{s}^{i}\right)$ where $\phi_{i}^{u}\left(k_{s}^{i}\right)$ is the control input signal at the latest triggering time $k_{s}^{i}$ and $\theta_{i}$ is a positive scalar to be designed. The executions are triggered as long as the condition

$$
\Upsilon_{i}\left(e_{i}(k), \theta_{i}\right)>0
$$

is satisfied. Obviously, the sequence of event triggering instants is determined iteratively by

$$
k_{s+1}^{i}=\inf \left\{k \in \mathbb{N} \mid k>k_{s}^{i}, \Upsilon_{i}\left(e_{i}(k), \theta_{i}\right)>0\right\}
$$

In the event-triggered setup, the control law can be further described by

$$
u_{i}(k)= \begin{cases}0, & k \in\left[0, k_{0}^{i}\right) \\ K \phi_{i}^{u}(k), & k \in\left[k_{0}^{i}, \infty\right)\end{cases}
$$

where $\phi_{i}^{u}(k)=0$ for $k \in\left[0, k_{0}^{i}\right)$ and $\phi_{i}^{u}(k)=\phi_{i}\left(k_{s}^{i}\right)$ for $k \in\left[k_{s}^{i}, k_{s+1}^{i}\right)$. It is easy to see that the controller input remains a constant in the execution interval $\left[k_{s}, k_{s+1}\right)$. Furthermore, such a control law can be rewritten as

$$
u_{i}(k)=K\left(\phi_{i}(k)+e_{i}(k)\right), \quad\left|e_{i}(k)\right|^{2} \leq \theta_{i} .
$$

The closed-loop system can be obtained as follows

$$
\begin{aligned}
x_{k+1}= & (I \otimes A+\mathcal{H} \otimes(B K C)) x_{k}+I \otimes D x_{k} w_{k} \\
& +\mathcal{H} \otimes(B K E) x_{k} v_{k}+I \otimes(B K) e_{k}
\end{aligned}
$$

where

$$
\begin{aligned}
& x_{k}=\left[\begin{array}{llll}
x_{1}^{T}(k) & \cdots & x_{N}^{T}(k)
\end{array}\right]^{T}, e_{k}=\left[\begin{array}{llll}
e_{1}^{T}(k) & \cdots & e_{N}^{T}(k)
\end{array}\right]^{T}, \\
& \mathcal{H}=\left[\begin{array}{cccc}
-\operatorname{deg}_{i n}^{1} & h_{1,2} & \cdots & h_{1, N} \\
\vdots & \vdots & \ddots & \vdots \\
h_{N, 1} & h_{N, 2} & \cdots & -\operatorname{deg}_{i n}^{N}
\end{array}\right] .
\end{aligned}
$$

On the other hand, denote the average state of all agents by

$$
\bar{x}_{k}=\frac{1}{N} \sum_{i=1}^{N} x_{i}(k)=\frac{1}{N}\left(\mathbf{1}^{T} \otimes I\right) x_{k} .
$$

Then, taking $\mathbf{1}^{T} \mathcal{H}=0$ into consideration, one has

$$
\bar{x}_{k+1}=A \bar{x}_{k}+D \bar{x}_{k} w_{k}+\frac{1}{N}\left(\mathbf{1}^{T} \otimes(B K)\right) e_{k} .
$$

Furthermore, in order to look at the deviation of each state from the average state, we denote $\tilde{x}_{i}(k):=x_{i}(k)-$ $\bar{x}_{k}$ and $\tilde{x}_{k}:=\left[\begin{array}{llll}\tilde{x}_{1}^{T}(k) & \tilde{x}_{2}^{T}(k) & \cdots & \tilde{x}_{N}^{T}(k)\end{array}\right]$. Moreover, subtracting (8) from (6) leads to

$$
\begin{aligned}
\tilde{x}_{k+1}= & (I \otimes A+\mathcal{H} \otimes(B K C)) \tilde{x}_{k}+I \otimes D \tilde{x}_{k} w_{k} \\
& +\mathcal{H} \otimes(B K E) \tilde{x}_{k} v_{k}+\mathcal{N} \otimes(B K) e_{k}
\end{aligned}
$$

where $\mathcal{N}=\left[a_{i j}\right]_{N \times N}$ with $a_{i j}=\frac{N-1}{N}$ for $i=j$ or $a_{i j}=$ $-\frac{1}{N}$ for $i \neq j$.

Similar to the method in [18], one can select $\varphi_{i} \in \mathbb{R}^{n}$ with $\varphi_{i}^{T} \mathcal{H}=\lambda_{i} \varphi_{i}^{T}(i=2,3, \cdots, N)$ to form the unitary matrix $M=\left[\mathbf{1} / \sqrt{N} \varphi_{2} \cdots \varphi_{N}\right]$ and then transform $\mathcal{H}$ into a diagonal form: $\operatorname{diag}\left\{0, \lambda_{2}, \cdots, \lambda_{N}\right\}=M^{T} \mathcal{H} M$. It can be found that $\varphi_{i}$ and $\lambda_{i}$ are the $i$ th eigenvector and eigenvalue of the Laplacian matrix $\mathcal{H}$. Furthermore, in this paper, we assume that eigenvalues of $\mathcal{H}$ in a descending order are written as $0=\lambda_{1}>\lambda_{2} \geq \lambda_{3} \geq \cdots \geq \lambda_{N}$. Define $\tilde{\eta}_{k}:=(M \otimes I)^{T} \tilde{x}_{k} \in \mathbb{R}^{n N}$ and partition it into two parts, i.e., $\tilde{\eta}_{k}=\left[\begin{array}{ll}\eta_{k}^{T} & \xi_{k}^{T}\end{array}\right]^{T}$, where $\eta_{k} \in \mathbb{R}^{n}$ is a vector consisting of the first $n$ elements of $\tilde{\eta}_{k}$. Due to $(\mathcal{H} \otimes(B K E))\left(\mathbf{1} \otimes \bar{x}_{k}\right)=0$, it can be easily shown that

$$
\left\{\begin{aligned}
\eta_{k+1}= & \frac{1}{\sqrt{N}} \sum_{i=1}^{N} \tilde{x}_{i}(k+1)=0 \\
\xi_{k+1}= & (\mathcal{A}+\Xi \otimes(B K C)) \xi_{k}+\Xi \otimes(B K E) \xi_{k} v_{k} \\
& +\mathcal{D} \xi_{k} w_{k}+(\mathcal{M N}) \otimes(B K) e_{k}
\end{aligned}\right.
$$

where $\Xi=\operatorname{diag}\left\{\lambda_{2}, \cdots, \lambda_{N}\right\}, \mathcal{M}=\left[\varphi_{2}^{T} \cdots \varphi_{N}^{T}\right]^{T} \mathcal{A}=$ $I_{N-1} \otimes A$ and $\mathcal{D}=I_{N-1} \otimes D$. 
Definition 1 Let the undirected communication graph $\mathscr{G}$, the parameter $\varepsilon>0$ and the bounded function $U\left(\left|\tilde{x}_{0}\right|\right)$ be given. The discrete-time stochastic multi-agent system (1) with the event-triggered control protocol (5) is said to reach the consensus with probability $1-\varepsilon$ if the system dynamics satisfies

$$
\operatorname{Prob}\left\{\left|x_{i}(k)-x_{j}(k)\right|<U\left(\left|\tilde{x}_{0}\right|\right)\right\} \geq 1-\varepsilon,
$$

for $\forall i, j \in \mathscr{V}, \forall k \geq 0$ and $\forall \tilde{x}_{0} \in \mathbb{R}^{n N} \backslash\{0\}$.

Definition 2 Let the undirected communication graph $\mathscr{G}$, the parameter $\varepsilon>0$ and the bounded function $U\left(\left|\tilde{x}_{0}\right|\right)$ be given. The discrete-time stochastic multi-agent system (1) is said to be $\varepsilon$-consensusable if there exist a controller gain $K$ in (2) and thresholds $\theta_{i}(i=1,2, \cdots, N)$ in the triggering condition (4) such that the closed-loop system (6) can reach the consensus with probability $1-\varepsilon$.

In this paper, we aim to design both the controller parameter $K$ and the threshold $\theta_{i}(i=1,2, \cdots, N)$ such that the discrete-time stochastic multi-agent system (1) reaches the consensus with probability $1-\varepsilon$.

Remark 1 Different from traditional consensus definitions focusing on the asymptotic (with time tending to infinity) behavior in the mean-square sense, the new consensus definition proposed in this paper is associated with the transient (at any time point $k$ ) behavior in the probability sense, that is, the consensus with probability $1-\varepsilon$. Our definition is more suitable when the transient and probabilistic performances of the consensus process are a concern, which is often the case in practice.

Remark 2 Another feature of this paper is that the threshold $\theta_{i}$ in (4) is a parameter to be designed and this provides more flexibility to achieve the desired consensus performance. Note that, in most existing literature, such kind of thresholds have been assumed to be fixed a priori. Obviously, the threshold of the triggered condition is closely related to the network traffic. A smaller threshold gives rise to a heavier network load, and therefore an adequate trade-off between the threshold and the acceptable network load.

\section{Main Results}

3.1 Input-to-state stability in probability for general discrete-time stochastic nonlinear systems

Consider the discrete-time stochastic nonlinear systems

$x_{k+1}=f\left(k, x_{k}, u_{k}\right)+g\left(k, x_{k}, u_{k}\right) w_{k}$

where $x_{k} \in \mathbb{R}^{n}$ is the state vector, $u_{k} \in \mathbb{R}^{p}$ is the control input, $w_{k} \in \mathbb{R}$ is a zero-mean random sequence with $\mathbb{E}\left\{w_{k}^{2}\right\}=1$, and $f, g:[0,+\infty) \times \mathbb{R}^{n} \times \mathbb{R}^{p} \rightarrow \mathbb{R}^{n}$ are two continuous functions satisfying $f(k, 0,0)=g(k, 0,0)=$ 0 for all $k \geq 0$.

First, let us introduce some important function classes. A function $\gamma: \mathbb{R}^{+} \rightarrow \mathbb{R}^{+}$is said to be of class $\mathscr{K}$ if it is a continuous strictly increasing function with $\gamma(0)=0$, and is said to be of class $\mathscr{K}_{\infty}$ if $\gamma \in \mathscr{K}$ with $\gamma(r) \rightarrow \infty$ as $r \rightarrow \infty$. Furthermore, a function $\beta: \mathbb{R}^{+} \times \mathbb{R}^{+} \rightarrow \mathbb{R}^{+}$is said to be of class $\mathscr{K} \mathscr{L}$ if the mapping $\beta(s, k)$ is of class $\mathscr{K}$ for each fixed $k$ and is decreasing to zero as $k \rightarrow \infty$ for each fixed $s$.

Definition 3 The system (12) is said to be input-tostate stable with probability if, for any $\varepsilon>0$, there exist functions $\hat{\beta} \in \mathscr{K} \mathscr{L}$ and $\gamma \in \mathscr{K}$ such that the system dynamic $x_{k}$ satisfies

$$
\operatorname{Prob}\left\{\left|x_{k}\right|<\hat{\beta}\left(\left|x_{0}\right|, k\right)+\gamma\left(\left\|u_{k}\right\|_{\infty}\right)\right\} \geq 1-\varepsilon
$$

for $\forall k \geq 0$ and $\forall x_{0} \in \mathbb{R}^{n} \backslash\{0\}$, where $\left\|u_{k}\right\|_{\infty}:=$ $\sup _{k}\left\{\left|u_{k}\right|\right\}$. In this case, the system (12) is said to have the input-to-state stability in probability (ISSiP).

For system (12), one has the following Lyapunov-like theorem.

Theorem 1 The stochastic system (12) is input-tostate stable with probability if there exist a positive definite function $\mathcal{V}:[0,+\infty) \times \mathbb{R}^{n} \rightarrow \mathbb{R}$ (called an ISSiP-Lyapunov function), two $\mathscr{K}_{\infty}$ class functions $\underline{\alpha}$ and $\bar{\alpha}$, and two $\mathscr{K}$ class functions $\chi$ and $\alpha$ such that, for all $x_{k} \in \mathbb{R}^{n} \backslash\{0\}$, the following two inequalities hold

$$
\begin{gathered}
\underline{\alpha}\left(\left|x_{k}\right|\right) \leq \mathcal{V}\left(k, x_{k}\right) \leq \bar{\alpha}\left(\left|x_{k}\right|\right), \\
\mathbb{E}\left\{\mathcal{V}\left(k+1, x_{k+1}\right) \mid \mathscr{F}_{k}\right\}-\mathcal{V}\left(k, x_{k}\right) \\
\leq \chi\left(\|u\|_{\infty}\right)-\alpha\left(\left|x_{k}\right|\right) .
\end{gathered}
$$

Furthermore, if (14) and (15) hold, then the functions $\hat{\beta}$ and $\gamma$ in Definition 3 can be selected as $\hat{\beta}(\cdot, k)=\sqrt{\underline{\alpha}^{-1}\left(\varepsilon^{-1} \phi^{k} \bar{\alpha}(\cdot)\right)}(0<\phi<1)$ and $\gamma=\sqrt{\underline{\alpha}\left(\varepsilon^{-1}\left(\bar{\alpha}\left(\alpha^{-1}(\chi(\cdot))+\chi(\cdot)\right)\right)\right.}$, respectively.

This theorem can be showed along the similar lines of the proofs of Theorem 2 in [9] and therefore the proof is omitted to the space saving.

\subsection{Event-triggered Consensus Control for Multi-agent Systems}

In Theorem 1, the relationship is described between the desired stability and the bound of inputs. For the eventtriggering mechanism proposed in this paper, when $e_{i}(k)$ overweighs a certain threshold $\theta_{i}$ for the system (1), the control input is updated and $e_{i}(k)$ returns to zero at the same time. As such, $e_{i}(k)$ is a bounded input and the stability result in Theorem 1 can be directly utilized to analyze the event-triggered consensus control problem.

Lemma 1 [8] Let $\Psi_{1}$ and $\Psi_{2} \in \mathbb{R}^{n \times n}$ be two symmetric matrices with their eigenvalues listed as follows

$\lambda_{1}\left(\Psi_{1}\right) \geq \cdots \geq \lambda_{n}\left(\Psi_{1}\right), \lambda_{1}\left(\Psi_{2}\right) \geq \cdots \geq \lambda_{n}\left(\Psi_{2}\right)$,

$\lambda_{1}\left(\Psi_{1}+\Psi_{2}\right) \geq \lambda_{2}\left(\Psi_{1}+\Psi_{2}\right) \geq \cdots \geq \lambda_{n}\left(\Psi_{1}+\Psi_{2}\right)$.

Then, we have $\lambda_{n}\left(\Psi_{1}+\Psi_{2}\right) \leq \lambda_{n}\left(\Psi_{1}\right)+\lambda_{1}\left(\Psi_{2}\right)$. Furthermore, if $\Psi_{1} \geq \Psi_{2}$, then $\lambda_{n}\left(\Psi_{1}\right) \geq \lambda_{n}\left(\Psi_{2}\right)$.

Theorem 2 Let the positive scalar $\varepsilon$ and the bounded function $U\left(\left|\tilde{x}_{0}\right|\right)$ be given. The discrete-time stochastic multi-agent system (1) with the undirected graph $\mathscr{G}$ and the event-triggered control law (5) is $\varepsilon$-consensusable if there exist a matrix $K$, two positive definite matrices $\mathcal{Q}$ and $\mathcal{P}$, and a positive scalar $\delta$ satisfying

$$
\begin{aligned}
\mathcal{Q}= & \mathcal{P}-(1+\delta)(\mathcal{A}+\Xi \otimes(B K C))^{T} \mathcal{P}(\mathcal{A}+\Xi \\
& \otimes(B K C))-\mathcal{D}^{T} \mathcal{P} \mathcal{D}-(\Xi \otimes(B K E))^{T} \mathcal{P} \\
& \times(\Xi \otimes(B K E))-\sigma^{2} \mathcal{D}^{T} \mathcal{P}(\Xi \otimes(B K E)) \\
& -\sigma^{2}(\Xi \otimes(B K E))^{T} \mathcal{P} \mathcal{D}
\end{aligned}
$$

and the following triggering condition

$$
\left|e_{i}(k)\right| \leq \theta_{i}:=\frac{\operatorname{deg}_{i n}^{i}\left(U\left(\left|\tilde{x}_{0}\right|\right)-2 \tilde{\beta}\left|\tilde{x}_{0}\right|\right)}{2 \tilde{\gamma} \sum_{j=1}^{N} \operatorname{deg}_{i n}^{j}}
$$


where

$$
\begin{aligned}
& \Gamma=\left(\delta^{-1}+1\right)(\mathcal{N} \otimes(B K))^{T} \mathcal{P}(\mathcal{N} \otimes(B K)) \\
& \tilde{\gamma}=\sqrt{\varepsilon^{-1} \lambda_{\min }^{-1}(\mathcal{P})\left(\lambda_{\max }(\mathcal{P}) \lambda_{\min }^{-1}(\mathcal{Q})+1\right) \lambda_{\max }(\Gamma)}, \\
& \tilde{\beta}=\sqrt{\varepsilon^{-1} \lambda_{\min }^{-1}(\mathcal{P}) \lambda_{\max }(\mathcal{P})} .
\end{aligned}
$$

Proof: Constructing the Lyapunov function $\mathcal{V}(k)=$ $\xi_{k}^{T} \mathcal{P} \xi_{k}$ and calculating its difference along the trajectory of system (10b), it follows that

$$
\begin{aligned}
& \mathbb{E}\{\Delta V(k)\}:=\mathbb{E}\left\{V(k+1)-V(k) \mid \xi_{k}\right\} \\
= & \xi_{k}^{T}\left((\mathcal{A}+\Xi \otimes(B K C))^{T} \mathcal{P}(\mathcal{A}+\Xi \otimes(B K C))\right. \\
& +\mathcal{D}^{T} \mathcal{P} \mathcal{D}+(\Xi \otimes(B K E))^{T} \mathcal{P}(\Xi \otimes(B K E)) \\
& \left.+2 \sigma^{2} \mathcal{D}^{T} \mathcal{P}(\Xi \otimes(B K E))-\mathcal{P}\right) \xi_{k} \\
& +2 \xi_{k}^{T}(\mathcal{A}+\Xi \otimes(B K C))^{T} \mathcal{P}((\mathcal{M N}) \otimes(B K)) e_{k} \\
& +e_{k}^{T}((\mathcal{M N}) \otimes(B K))^{T} \mathcal{P}((\mathcal{M N}) \otimes(B K)) e_{k} .
\end{aligned}
$$

Considering $\mathcal{M}^{T} \mathcal{M}=I$ and the elementary inequality $2 a^{T} b \leq \delta a^{T} a+\delta^{-1} b^{T} b$, we have

$$
\begin{aligned}
\mathbb{E}\{\Delta V(k)\} & \leq-\xi_{k}^{T} \mathcal{Q} \xi_{k}+e_{k}^{T} \Gamma e_{k} \\
& \leq-\lambda_{\min }(\mathcal{Q})\left|\xi_{k}\right|^{2}+\lambda_{\max }(\Gamma)\left\|e_{k}\right\|_{\infty}^{2} .
\end{aligned}
$$

Selecting $\chi\left(\|e\|_{\infty}\right)=\lambda_{\max }(\Gamma)\left\|e_{k}\right\|_{\infty}^{2}$ and $\alpha\left(\left|\xi_{k}\right|\right)=$ $\lambda_{\min }(Q)\left|\xi_{k}\right|^{2}$, it follows from Theorem 1 that the system modeled by (10a) and (10b) is input-to-state stable with probability. Since $\tilde{\eta}_{k}=(M \otimes I)^{T} \tilde{x}_{k}$ and $(M \otimes I)(M \otimes I)^{T}=I$, we have

$$
\begin{aligned}
& \operatorname{Prob}\left\{\left|\xi_{k}\right| \leq \beta\left(\left|\xi_{0}\right|, 0\right)+\gamma\left(\left\|e_{k}\right\|_{\infty}\right)\right\} \\
= & \operatorname{Prob}\left\{\left|(M \otimes I)^{T} \tilde{x}_{k}\right| \leq \beta\left(\left|(M \otimes I)^{T} \tilde{x}_{0}\right|, 0\right)\right. \\
& \left.+\gamma\left(\left\|e_{k}\right\|_{\infty}\right)\right\} \\
\geq & \operatorname{Prob}\left\{\left|\tilde{x}_{k}\right| \leq \hat{\beta}\left(\left|\tilde{x}_{0}\right|, k\right)+\gamma\left(\left\|e_{k}\right\|_{\infty}\right)\right\} \\
\geq & 1-\varepsilon, \quad \forall \tilde{x}_{0} \in \mathbb{R}^{n N} \backslash\{0\}
\end{aligned}
$$

where $\beta(z, 0)=\tilde{\beta} z$ and $\gamma(z)=\tilde{\gamma} z$ for $\forall z \in \mathbb{R}$.

On the other hand, in terms of the triggering condition (17), one has

$$
\left|e_{k}\right| \leq\left(U\left(\left|\tilde{x}_{0}\right|\right)-2 \tilde{\beta}\left|\tilde{x}_{0}\right|\right) / 2 \tilde{\gamma} .
$$

In addition, it is easy to see that

$$
\left|x_{i}(k)-x_{j}(k)\right| \leq 2\left|\tilde{x}_{k}\right| .
$$

Finally, it follows from (20)-(22) that

$$
\begin{aligned}
& \operatorname{Prob}\left\{\left|x_{i}(k)-x_{j}(k)\right| \leq U\left(\left|\tilde{x}_{0}\right|\right)\right\} \\
\geq & \operatorname{Prob}\left\{\left|\tilde{x}_{k}\right| \leq \beta\left(\left|\tilde{x}_{0}\right|, 0\right)+\gamma\left(\left\|e_{k}\right\|_{\infty}\right)\right\} \geq 1-\varepsilon
\end{aligned}
$$

for $\forall i, j \in \mathscr{V}$, which completes the proof.

In what follows, a sufficient condition is provided in order to reduce the computation complexity by using the second and $N$ th eigenvalues of $\mathcal{H}$.

Theorem 3 Let the positive scalar $\varepsilon$ and the bounded function $U\left(\left|\tilde{x}_{0}\right|\right)$ be given. The discrete-time stochastic multi-agent system (1) with the undirected graph $\mathscr{G}$ and the event-triggered control law (5) is $\varepsilon$-consensusable, if there exist a matrix $K$, positive definite matrix $P$, and two positive scalars $\delta$ and $\varpi$ satisfying

a) when $\lambda_{2} \neq \lambda_{N}$

$$
\left\{\begin{aligned}
(1 & \left.\left.-\sigma^{4}\right) D^{T} P D-P+(1+\delta)\left(A+\lambda_{2} B K C\right)\right)^{T} \\
& \times P\left(A+\lambda_{2} B K C\right)+\varpi\left(\lambda_{2}-\lambda_{N}\right) I+\left(\sigma^{2} D\right. \\
& \left.+\lambda_{2} B K E\right)^{T} P\left(\sigma^{2} D+\lambda_{2} B K E\right)<0 \\
(1 & +\delta)\left((B K C)^{T} P A+A^{T} P B K C+\left(\lambda_{2}+\lambda_{N}\right)(B K\right. \\
& \left.\times C)^{T} P B K C\right)+\varpi I+\left(\sigma^{2}(B K E)^{T} P D+\sigma^{2} D^{T}\right. \\
& \left.\times P B K E+\left(\lambda_{2}+\lambda_{N}\right)(B K E)^{T} P B K E\right)>0(23 \mathrm{~b})
\end{aligned}\right.
$$

b) when $\lambda_{2}=\lambda_{N}$

$$
\begin{aligned}
(1 & \left.-\sigma^{4}\right) D^{T} P D-P+(1+\delta)(A \\
& \left.\left.+\lambda_{2} B K C\right)\right)^{T} P\left(A+\lambda_{2} B K C\right) \\
& +\left(\sigma^{2} D+\lambda_{2} B K E\right)^{T} P\left(\sigma^{2} D+\lambda_{2} B K E\right)<0
\end{aligned}
$$

and the triggering condition

$$
\left|e_{i}(k)\right| \leq \theta_{i}:=\frac{\operatorname{deg}_{i n}^{i}\left(U\left(\left|\tilde{x}_{0}\right|\right)-2 \bar{\beta}\left|\tilde{x}_{0}\right|\right)}{2 \bar{\gamma} \sum_{j=1}^{N} d e g_{i n}^{j}},
$$

where

$$
\begin{aligned}
Q_{2}= & P-\left(1-\sigma^{4}\right) D^{T} P D-(1+\delta)(A \\
& \left.\left.+\lambda_{2} B K C\right)\right)^{T} P\left(A+\lambda_{2} B K C\right) \\
& -\left(\sigma^{2} D+\lambda_{2} B K E\right)^{T} P\left(\sigma^{2} D+\lambda_{2} B K E\right), \\
\Gamma= & \left(\delta^{-1}+1\right)((\mathcal{M N}) \otimes(B K))^{T} \\
& \times(I \otimes P)((\mathcal{M N}) \otimes(B K)), \\
\bar{\beta}= & \sqrt{\varepsilon^{-1} \lambda_{\min }^{-1}(P) \lambda_{\max }(P)}, \\
\pi= & \lambda_{\min }\left(Q_{2}\right)-\varpi\left(\lambda_{2}-\lambda_{N}\right)>0, \\
\bar{\gamma}= & \sqrt{\varepsilon^{-1} \lambda_{\min }^{-1}(P)\left(q \pi^{-1} \lambda_{\max }(P)+1\right) \lambda_{\max }(\Gamma) .}
\end{aligned}
$$

Proof: Based on Theorem 2, it suffices to show that (16)(17) are satisfied for both cases of $\lambda_{2} \neq \lambda_{N}$ and $\lambda_{2}=\lambda_{N}$.

Case a) $\lambda_{2} \neq \lambda_{N}$. First, it follows from (23a) that $Q_{2}>$ $\bar{\varpi}\left(\lambda_{2}-\lambda_{N}\right) I$. For $1<i<N$, denote

$$
\begin{aligned}
Q_{i}= & P-\left(1-\sigma^{4}\right) D^{T} P D-(1+\delta)(A \\
& \left.\left.+\lambda_{i} B K C\right)\right)^{T} P\left(A+\lambda_{i} B K C\right) \\
& -\left(\sigma^{2} D+\lambda_{i} B K E\right)^{T} P\left(\sigma^{2} D+\lambda_{i} B K E\right) .
\end{aligned}
$$

As $\lambda_{N}<\lambda_{i}=\lambda_{2}(1<i<N)$, one has $Q_{i}=Q_{2}$. Furthermore, it follows from $\lambda_{N}<\lambda_{i}<\lambda_{2}<0$ that

$$
\begin{aligned}
& Q_{i}-Q_{2}+\varpi\left(\lambda_{2}-\lambda_{N}\right) I \\
\geq & \left(\lambda_{2}-\lambda_{i}\right)(1+\delta)\left((B K C)^{T} P A+A^{T} P B K C+\left(\lambda_{2}\right.\right. \\
& \left.\left.+\lambda_{N}\right)(B K C)^{T} P B K C\right)+\left(\lambda_{2}-\lambda_{i}\right)\left(\sigma^{2}(B K E)^{T}\right. \\
& \times P D+\sigma^{2} D^{T} P B K E+\left(\lambda_{2}+\lambda_{N}\right)(B K E)^{T} P \\
& \times B K E+\varpi I)+\left(\lambda_{2}-\lambda_{i}\right)\left(\left(\lambda_{2}+\lambda_{i}\right)-\left(\lambda_{N}+\lambda_{2}\right)\right) \\
& \times\left((1+\delta)(B K C)^{T} P B K C+(B K E)^{T} P B K E\right) \\
> & 0
\end{aligned}
$$


which implies $Q_{i} \geq Q_{2}-\varpi\left(\lambda_{2}-\lambda_{N}\right) I>0$. Therefore, by selecting $\Psi_{1}=Q_{i}, \Psi_{2}=\varpi\left(\lambda_{2}-\lambda_{N}\right) I$ and exploiting Lemma 1 , it can be found that

$$
\begin{aligned}
\lambda_{\min }\left(Q_{i}\right) & +\lambda_{\max }\left(\varpi\left(\lambda_{2}-\lambda_{N}\right) I\right) \\
& \geq \lambda_{\min }\left(Q_{i}+\varpi\left(\lambda_{2}-\lambda_{N}\right)\right) \geq \lambda_{\min }\left(Q_{2}\right)
\end{aligned}
$$

which results in

$$
\lambda_{\min }\left(Q_{i}\right) \geq \lambda_{\min }\left(Q_{2}\right)-\varpi\left(\lambda_{2}-\lambda_{N}\right)
$$

and therefore

$$
\begin{aligned}
\lambda_{\min }(\mathcal{Q}) & :=\lambda_{\min }\left(\operatorname{diag}\left\{Q_{2}, Q_{3}, \cdots, Q_{N}\right\}\right) \\
& \geq \lambda_{\min }\left(Q_{2}\right)-\varpi\left(\lambda_{2}-\lambda_{N}\right)>0 .
\end{aligned}
$$

By selecting $\mathcal{P}=I \otimes P$, it can be easily checked that (17) in Theorem 2 can be guaranteed.

On the other hand, in terms of (28) and the selected $\mathcal{P}$, one has $\bar{\beta}=\tilde{\beta}$ and $\bar{\gamma}>\tilde{\gamma}$. Therefore, it follows that

$$
\left|e_{i}(k)\right| \leq \frac{\operatorname{deg}_{\text {in }}^{i}\left(U\left(\left|\tilde{x}_{0}\right|\right)-2 \tilde{\beta}\left|\tilde{x}_{0}\right|\right)}{2 \tilde{\gamma} \sum_{j=1}^{N} \operatorname{deg}_{\text {in }}^{j}}
$$

and therefore (16) in Theorem 2 holds.

Case b) $\lambda_{2}=\lambda_{N}$. Using the similar procedure in the proof of Case a), one can find from (24) and (26) that $Q_{N}=Q_{N-1}=\cdots=Q_{2}>0$. Therefore, $\lambda_{\min }(\mathcal{Q})=$ $\lambda_{\min }\left(Q_{2}\right)>0$ which, in turn, implies that (16)-(17) in Theorem 2 are true.

So far, it can be concluded that, for both Case a) and Case b), (16)-(17) are satisfied and it then follows from Theorem 2 that the discrete-time stochastic multi-agent system (1) is $\varepsilon$-consensusable.

Remark 3 It is worth pointing out that the selected functions $\beta(\cdot, \cdot)$ and $\gamma(\cdot)$ to obtain the desired result are rough but possess the advantage of convenient implementation. Such a selected strategy could reduce the feasibility of the controller design scheme as the consensus probability increases. So, in order to improve the feasibility of design scheme, how to design the new functions $\beta(\cdot, \cdot)$ and $\gamma(\cdot)$ to reduce the conservatism is the further topic to be investigated.

Having obtained the analysis results, we are now in a position to handle the design problem of both the threshold $\theta_{i}$ and the controller gain matrix $K$. First, in terms of the Schur Complement Lemma, (23a), (23b) and (24) are, respectively, equivalent to

$$
\begin{aligned}
& {\left[\begin{array}{ccc}
\Pi_{11}+\varpi\left(\lambda_{2}-\lambda_{N}\right) I & * & * \\
\tilde{\delta} \Pi_{21} & -P^{-1} & * \\
\Pi_{31} & 0 & -P^{-1}
\end{array}\right]<0} \\
& {\left[\begin{array}{ccc}
\bar{\Pi}_{11} & * & * \\
\tilde{\delta} \bar{\Pi}_{21} & -P^{-1} & * \\
\bar{\Pi}_{31} & 0 & -P^{-1}
\end{array}\right]<0} \\
& {\left[\begin{array}{ccc}
\Pi_{11} & * & * \\
\tilde{\delta} \Pi_{21} & -P^{-1} & * \\
\Pi_{31} & 0 & -P^{-1}
\end{array}\right]<0}
\end{aligned}
$$

where

$$
\begin{aligned}
& \Pi_{11}=\left(1-\sigma^{4}\right) D^{T} P D-P, \Pi_{21}=A+\lambda_{2} B K C, \\
& \Pi_{31}=\sigma^{2} D+\lambda_{2} B K E, \quad \bar{\lambda}=\sqrt{-\left(\lambda_{2}+\lambda_{N}\right)}, \\
& \bar{\Pi}_{11}=-\omega I-\tilde{\delta} \bar{\lambda}^{-2} A^{T} P A-\sigma^{4} \bar{\lambda}^{-2} D^{T} P D, \\
& \bar{\Pi}_{21}=\bar{\lambda}^{-1} A-\bar{\lambda} B K C, \quad \tilde{\delta}=\sqrt{1+\delta}, \\
& \bar{\Pi}_{31}=\sigma^{2} \bar{\lambda}^{-1} D-\bar{\lambda} B K E .
\end{aligned}
$$

Similar to the method in [5], by introducing a free matrix

$$
R:=\left[\begin{array}{cc}
R_{11} & R_{12} \\
0 & R_{22}
\end{array}\right]
$$

where $R_{11} \in \mathbb{R}^{p \times p}, R_{12} \in \mathbb{R}^{n \times(n-p)}$ and $R_{22} \in$ $\mathbb{R}^{(n-p) \times(n-p)}$ are arbitrary matrices. Then, denote $W=$ $\left[B\left(\left(B^{T} B\right)^{-1}\right)^{T} B^{\perp}\right]^{T}, \tilde{K}:=\left[\begin{array}{ll}\bar{K}^{T} & 0\end{array}\right]^{T}:=R W B K$ and $\Omega:=P-R W-W^{T} R^{T}$ where $B^{\perp}$ stands for an orthogonal basis of the null space for $B^{T}$. We conclude that (30) is satisfied if

$$
\begin{aligned}
& {\left[\begin{array}{ccc}
\Pi_{11}+\varpi\left(\lambda_{2}-\lambda_{N}\right) I & * & * \\
\tilde{\delta}\left(R W A+\lambda_{2} \tilde{K} C\right) & \Omega & * \\
\sigma^{2} R W D+\lambda_{2} \tilde{K} E & 0 & \Omega
\end{array}\right]<0,} \\
& {\left[\begin{array}{ccc}
\bar{\Pi}_{11} & * & * \\
\tilde{\delta}\left(\bar{\lambda}^{-1} R W A-\bar{\lambda} \tilde{K} C\right) & \Omega & * \\
\sigma^{2} \bar{\lambda}^{-1} R W D-\bar{\lambda} \tilde{K} E & 0 & \Omega
\end{array}\right]<0,} \\
& {\left[\begin{array}{ccc}
\Pi_{11} & * & * \\
\tilde{\delta}\left(R W A+\lambda_{2} \tilde{K} C\right) & \Omega & * \\
\sigma^{2} R W D+\lambda_{2} \tilde{K} E & 0 & \Omega
\end{array}\right]<0 .}
\end{aligned}
$$

Theorem 4 Let the positive scalar $\varepsilon$ and the bounded function $U\left(\left|\tilde{x}_{0}\right|\right)$ be given. The discrete-time stochastic multi-agent system (1) with the undirected graph $\mathscr{G}$ and the event-triggered control law (5) is $\varepsilon$-consensusable if there exist two matrices $\tilde{K}$ and $R$, positive definite matrix $P$, and positive scalars $\varpi$ and $\tilde{\delta}>1$ satisfying: a) (32) and (33) when $\lambda_{2} \neq \lambda_{N}$, and b) (34) when $\lambda_{2}=\lambda_{N}$ where the related parameters are defined as in (31) and Theorem 3. Furthermore, if (32)-(33) hold for Case a) or (34) holds for Case b), then the output feedback controller gain matrix is given by $K=R_{11}^{-1} \tilde{K}$ and the threshold $\theta_{i}$ of the triggering condition (4) is determined by (25).

\section{A Simulation Example}

In this section, a simulation example is presented to illustrate the effectiveness of the proposed event-triggered consensus protocol for discrete-time stochastic multiagent systems with state-dependent noise.

Consider the system (1) with

$$
\begin{aligned}
& A=\left[\begin{array}{cc}
1 & 0.1 \\
0 & 1
\end{array}\right], B=\left[\begin{array}{l}
0.20 \\
0.25
\end{array}\right], D=\left[\begin{array}{cc}
0 & 0.01 \\
0 & 0
\end{array}\right], \\
& C=\left[\begin{array}{ll}
-0.3 & 0
\end{array}\right], E=\left[\begin{array}{ll}
0.01 & 0
\end{array}\right] .
\end{aligned}
$$

Suppose that there are five agents with an undirected communication graph $\mathcal{G}$ where the associated adjacency matrix $\mathcal{H}$ is selected as follows 


$$
\mathcal{H}=\left[\begin{array}{ccccc}
-6 & 3 & 0 & 0 & 3 \\
3 & -6 & 3 & 0 & 0 \\
0 & 3 & -6 & 3 & 0 \\
0 & 0 & 3 & -6 & 3 \\
3 & 0 & 0 & 3 & -6
\end{array}\right] .
$$

For such a Laplacian matrix, the eigenvalues are $\lambda_{2}=$ $\lambda_{3}=-4.1459, \lambda_{4}=\lambda_{5}=-10.8541$. Select $U\left(\left|\tilde{x}_{0}\right|\right)=$ $15\left|\tilde{x}_{0}\right|, \sigma^{2}=0.0025$ and $\varepsilon=0.25$. By linearly searching $\tilde{\delta}$ over the interval [1.01 2 [ ] and solving the resulting linear matrix inequalities by using the Matlab software (with the YALMIP 3.0, Version No. R20100813) where the solver is selected as 'solvesdp', a set of feasible solutions is obtained as follows:

$P=\left[\begin{array}{cc}3.9692 & -1.5969 \\ -1.5969 & 1.2032\end{array}\right], \quad R=\left[\begin{array}{cc}0.4056 & -13.0834 \\ 0 & 6.0227\end{array}\right]$,

$\tilde{K}=-0.4051, \quad \varpi=8.0871 \times 10^{-4}, \quad \tilde{\delta}=1.15$

and therefore $K=-0.9987, \lambda_{\max }(P)=4.6988$, $\lambda_{\min }(P)=0.4737, \lambda_{\min }\left(Q_{2}\right)=0.0428, \lambda_{\max }(\Gamma)=$ $0.5680, \tilde{\beta}=6.22992$ and $\bar{\gamma}=16.9672$.

In the simulation, $x_{s}(0)(s=1,2, \cdots, 5)$ are generated that obey Gaussian distribution $\mathcal{N}\left(\hat{x}_{0}^{s}, \bar{\Lambda}^{s}\right)$ with $\hat{x}_{0}^{s}=$ $\left[\begin{array}{ll}0.6+0.1 s & 0.8+0.13 s\end{array}\right]^{T}$ and $\bar{\Lambda}^{s}=\operatorname{diag}\{0.25+0.1 s, 0.15+$ $0.08 s\}$. According to the inequality (25), the triggering condition is $\left|e_{s}\right|>0.0257(s=1,2, \cdots, 5)$.

Simulation results are shown in Figs. $1 \sim 3$. Figs. 1 and 2 plot the state trajectories of $x_{s}(k)(s=1,2, \cdots, 5)$ for the discrete-time stochastic multi-agent system (1), respectively, with and without the event-triggered consensus controller (5). Obviously, the open-loop multiagent system cannot reach the desired consensus and the closed-loop one does so. Furthermore, the update times are shown in Fig. 3, from which we can easily find that the proposed consensus control scheme can effectively reduce the update frequency as compared with the traditional time-triggered mechanism. The simulation results have confirmed that the designed event-triggered consensus controller performs very well.

\section{Conclusions}

In this paper, the event-triggered consensus control problem has been addressed for a class of discrete-time stochastic multi-agent systems with state-dependent noises. First, a novel definition of consensus in probability has been provided which can be employed to characterize the dynamics of the consensus process. Then, by using intensive stochastic analysis approaches, a discrete-time version of the stability analysis results for ISSiP has been derived for a class of general discrete-time stochastic nonlinear systems. Within the established discrete-time framework, some sufficient conditions have been established for the existence of the desired event-triggered consensus controllers.

\section{References}

[1] F. Abdollahi and K. Khorasani, A decentralized Markovian jump $\mathcal{H}_{\infty}$ control routing strategy for mobile multi-agent networked systems, IEEE Transactions on Control Systems Technology, vol. 19, no. 2, pp. 269-283, Mar. 2011.

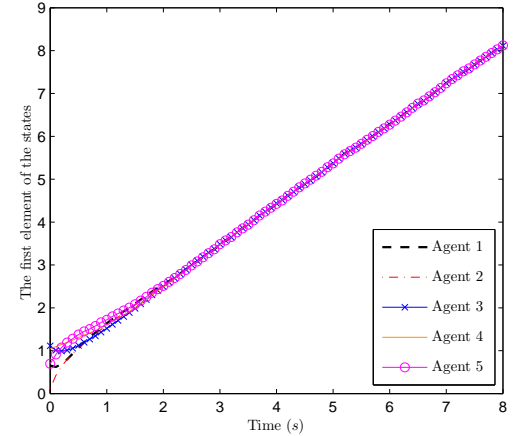

(a) The first element of the state $x_{s}(k)$

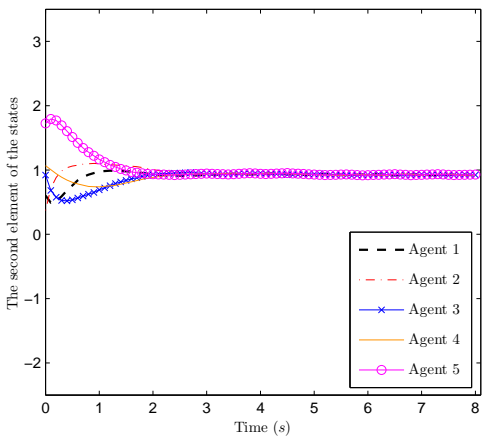

(b) The second element of the state $x_{s}(k)$

Fig. 1. The state trajectories of the closed-loop system.

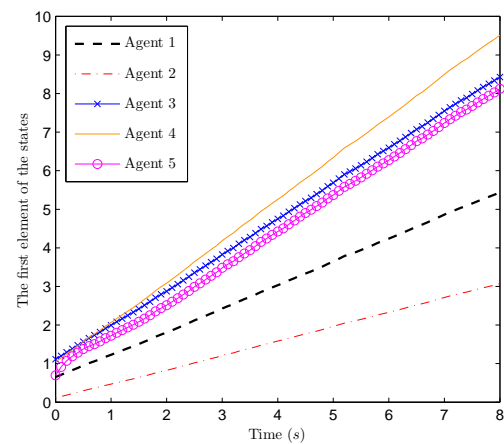

(a) The first element of the state $x_{s}(k)$

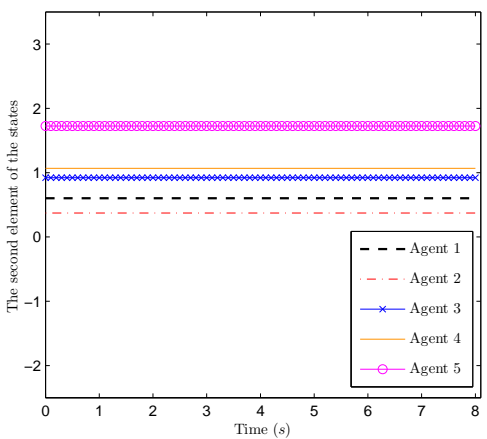

(b) The second element of the state $x_{s}(k)$

Fig. 2. The state trajectories of the open-loop system. 


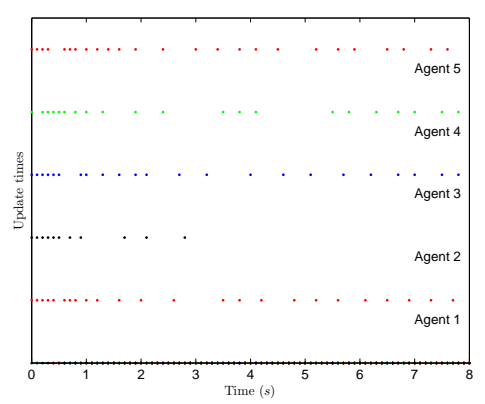

Fig. 3. Update times of controllers.

[2] D. Ding, Z. Wang, J. Lam and B. Shen, Finitehorizon $\mathcal{H}_{\infty}$ control for discrete time-varying systems with randomly occurring nonlinearities and fading measurements, IEEE Transactions on Automatic Control, 2015. DOI 10.1109/TAC.2014.2380671.

[3] D. Ding, Z. Wang, B. Shen and H. Dong, Envelopeconstrained $\mathcal{H}_{\infty}$ filtering with fading measurements and randomly occurring nonlinearities: The finite horizon case, Automatica, vol. 55, pp. 37-45, May 2015.

[4] H. Dong, Z. Wang, S. X. Ding and H. Gao, Finite-horizon estimation of randomly occurring faults for a class of nonlinear time-varying systems, Automatica, vol. 50, no. 12, pp. 3182-3189, Dec. 2014.

[5] J. Dong and G.-H. Yang, Robust static output feedback control for linear discrete-time systems with time-varying uncertainties, Systems and Control Letters, vol. 57, no. 2, pp. 123-131, Feb. 2008.

[6] T. Henningsson, Event-based control and estimation with stochastic disturbances, Thesis, Lund University Press, ISSN 0280-5316, 2008.

[7] A. Hu, J. Cao, M. Hua, and L. Guo, Event-triggered consensus of multi-agent systems with noises, Journal of the Franklin Institute, 2014, doi:10.1016/j.jfranklin.2014.08.005.

[8] P. Lancaster and M. T. Tismenetsky, The theory of matrices: with applications, Academic, New York, USA, 1985.

[9] S. Liu and J. Zhang, Output-feedback control of a class of stochastic nonlinear systems with linearly bounded unmeasurable states, International Journal of Robust and Nonlinear Control, vol. 18, no. 6, pp. 665-687, Apr. 2008.

[10] M. Mazo, A. Anta, and Paulo Tabuada, An ISS self-triggered implementation of linear controllers, Automatica, vol. 46, no. 8, pp. 1310-1314, Aug. 2010.

[11] X. Meng and T. Chen, Event based agreement protocols for multi-agent networks, Automatica, vol. 49, no. 7, pp. 21252132, Jul. 2013.

[12] W. Ren, K. Moore, and Y. Chen, High-order consensus algorithms in cooperative vehicle systems, Proceedings of the IEEE International Conference on Networking, Sensing and Control, Fort Lauderdale, U.S.A., pp. 457-462, 2006.

[13] G. S. Seyboth, D. V. Dimarogonas, and K. H. Johansson, Event-based broadcasting for multi-agent average consensus, Automatica, vol. 49, no. 1, pp. 245-252, Jan. 2013.

[14] J. Tsinias, The concept of 'Exponential input to state stability' for stochastic systems and applications to feedback stabilization, Systems and Control Letters, vol. 36, no. 3, pp. 221-229, Mar. 1999.

[15] X. Wang, E. E. Yaz, J. Long, Robust and resilient statedependent control of discrete-time nonlinear systems with general performance criteria, Systems Science \& Control Engineering, An Open Access Journal, vol. 2, no. 1, pp. 48$54,2014$.

[16] G. Wei, S. Liu, Y. Song and Y. Liu, Probability-guaranteed set-membership filtering for systems with incomplete measurements, Automatica, vol. 60, pp. 12-16, Oct. 2015.
[17] X. Yin and D. Yue, Event-triggered tracking control for heterogeneous multi-agent systems with Markov communication delays, Journal of the Franklin Institute, vol. 350, no. 3, pp. 1312-1334, Apr. 2013.

[18] K. You and L. Xie, Coordination of discrete-time multi-agent systems via relative output feedback, International Journal of Robust and Nonlinear Control, vol. 21, no. 13, pp. 15871605, Sep. 2011.

[19] W. Zhu, Z.-P. Jiang, and G. Feng, Event-based consensus of multi-agent systems with general linear models, Automatica, vol. 50, no. 2, pp. 552-558, Feb. 2014 